\title{
Physicians' View on Resuscitation Discussions among Patients and their Family in Comprehensive Cancer Center
}

\author{
Abdullah Bany Hamdan ${ }^{1}$, Sami Ayed AlShammary ${ }^{1}$, Jesusa Christine Tamani ${ }^{1}$, Sreemol \\ Sashidharan $^{1}$, Savithiri Ratnapalan ${ }^{2}$, Musa AlHarbi ${ }^{1}$ \\ ${ }^{1}$ Comprehensive Cancer Center, King Fahad Medical City, Riyadh, Saudi Arabia \\ ${ }^{2}$ Department of Pediatrics, Dalla Lana School of Public Health, University of Toronto, Canada \\ *Corresponding Author: Abdullah Bany Hamdan, Comprehensive Cancer Center, King Fahad \\ Medical City, Riyadh, Saudi Arabia
}

\begin{abstract}
Cardiopulmonary resuscitation can be medically futile and lead to prolonged suffering in some patients with disseminated malignancies. The aim of this study is to identify physicians' awareness, knowledge, and attitudes towards performing or withholding Cardiopulmonary resuscitation in cancer patients in the Middle East and the process they preferred for discussing this with the patients and families. A crosssectional survey was used and sent to physicians from different specialties in Comprehensive Cancer Center. There was a 68\% ( 51 out of 75 ) response rate for the survey; the majority(82\%) were males. $84 \%$ identified the discussion of resuscitation with patients and relatives as important, and 98\% stated that they would prefer not to resuscitate patients with metastatic disease where the resuscitation would be medically futile. More than half $57 \%$ wished to discuss resuscitation with both patients and relatives, together and $12 \%$ (6) stated that patients alone should be involved in this discussion. The best time for resuscitation discussion is when the patient is conscious and oriented by $86 \%(n=43)$ of responders; $10.0 \%(n=5))$ wished to wait till the patient was comatose and requirement of resuscitative measures were imminent. Less than half the responders (43\%) were aware of hospital policies regarding resuscitation. Most physicians in our study were aware of the importance of resuscitation discussion in cancer patients but varied in their approach as to when and to whom they should have these discussions. A small minority still believed that resuscitation should continue regardless of medical futility and most were not aware of hospital policies. This study highlights the importance of education and further training in the discussion about cardiopulmonary resuscitation for physicians involved in cancer care.
\end{abstract}

Keywords: Resuscitation, Oncology, Do-Not-Resuscitate, Attitude, Knowledge, Decision-making

\section{INTRODUCTION}

The rate of survival following Cardiopulmonary Resuscitation (CPR) depends on several factors which include but is not limited to: the severity of illness, type of chronic health condition, comorbidities, functional dependence and the number of CPR attempts made. Some cancer patients, most especially those with metastatic progression, may have lower survival rates after CPR.

In worst cases, there may even be a potential harm if patients with fatal and irreversible diseases are kept on a full-code status, undergoing painful and dehumanizing procedures like chest compressions, intubations, and mechanical ventilations, even when their clinical conditions imply that resuscitation efforts will not bring 
Physicians' View on Resuscitation Discussions among Patients and their Family in Comprehensive Cancer Center

any comfort or medical benefit for them. In these cases, Do-Not-Resuscitate (DNR) order is indicated for them wherein the goal is diverted to providing support for the patient and maximizing their optimum level of functioning rather than giving false hopes for survival.

Physicians are responsible for decision-making for DNR status, and they need to be aware of the patients' and family's concerns and ethical aspects of performing and not performing CPR. "The DNR order is carried out only to withhold resuscitation when a cardiopulmonary arrest occurs." However, some heath care providers fail to discuss with patients regarding changes related to DNR status [1].

There may be many differences in performing care for patients with DNR status. Some of these are related to legal issues, morality concepts, and appropriateness in medical instructions. The process for decision-making for this subject considered as an elite extent assigned for physicians. However, the physicians face many issues on decisionmaking process as it is related to many ethical aspects. There are significant changes in doctors' attitude for decision making, implementation and instructions for DNR due to a diversity of views due to culture, religion and geographical locations resulting in a lower rate of DNR orders in Asian and Muslim countries compared to the European countries [1].

DNR discussions with patients and family may cause stress among physicians, and it could be physical, mental, or emotional reactions [2, 3]. Anxiety and stress affect both physicians health, and patients care, especially in cardiopulmonary resuscitation (CPR) were quick and appropriate sequences of actions needed in a high-stress situation. Attending to improper cardiac arrest calls and communicating with very ill patients may lead to stress [2].
To reduce inappropriate $\mathrm{CPR}$ and poor outcome, senior physicians must ensure adequate review and documentation of the DoNot-Resuscitate order where appropriate, after discussion with patients and family. The quality of CPR may differ among hospitals within the same country, reflecting significant diversities among attitudes and performance of healthcare providers involved in CPR. The attitudes of health professionals may be affected by factors, such as conscience, personal experience, and education [4].

Ethical issues concerning resuscitation are increasingly important. Policies and guidelines support cardiopulmonary resuscitation will help health care workers for effective implementation. Although the chain of survival as described by American Heart Association has undoubtedly improved survival for patients who have an out of hospital cardiac arrest, overall survival remains between $1 \%$ and $25 \%$ [5].

A physician with different level of training is entrusted with the responsibility for the decision making. It is either for CPR or for do not resuscitate order where ethical and legal issues must be considered in addition to the chances of survival when there is no DNR discussion prior to an arrest in a patient where CPR may be medically futile.

Medical decisions should be made jointly by patient and physician, based on the patient goals, prognostic information, and the physician's expertise [6]. This is essential for palliative decisions; especially the patient care goal may be focused to provide comfort rather than longevity. A physician should consider about patients and families preferences for end of life care or resuscitation. The doctor's opinions toward DNR should be in agreement with medical ethics and institutional policies, protecting the best interests of the patient to providing excellent quality care [1]. 
Physicians' View on Resuscitation Discussions among Patients and their Family in Comprehensive Cancer Center

Continuous reinforcement is one of the key strategies for implementing practice change in resuscitation [7]. It is also necessary to recognize that language between doctor and patient is constrained by cultural norms especially when communicating bad news [8].

The different interpretations of DNR orders are a matter of concern in that they may affect patient treatment [9]. The awareness of health care professionals that Inaccurate beliefs about cardiopulmonary resuscitation can adversely impact on decision-making about resuscitation by older patients is important when discussion DNR [10].

The ethical, moral and legal implications of decisions to withhold and withdraw life support are not straight forward. Making the end of life decisions especially when the cultural background of doctor and patient differ, become even more challenging [11]. It has been shown that DNR order recommendations vary with the medical specialty, years of training and experience [12]. There are insufficient literatures regarding the attitudes and beliefs of patients, families, and health care professionals in different regions of the world [13]. This research aims to study physicians' awareness about CPR and their extent of knowledge as to when should patient or family will be informed about the need for resuscitation or the indication for DNR status for cancer patients in the Saudi Arabia.

\section{METHOdoloGY}

The researchers conducted a cross-sectional survey among physicians in different Specialties: Radiation Oncology, Palliative Department, Adult Medical Oncology, Adult Hematology, and Pediatric Hematology and Oncology in a tertiary care hospital in the Middle East. The survey tool consisted of two different parts: The first part elicited their demographic data while the second part aimed to obtain their attitude towards resuscitation. The questionnaire was administered in English.
Physicians (Consultants, Assistant Consultants, Fellows, and Residents) working in Comprehensive Cancer Center Adult and Pediatric Units of King Fahad Medical City, Saudi Arabia were invited to participate in the study. The study period was from August to September 2016.

The survey was sent to 75 physicians once, and 51 physicians consented to the study and responded.

There was no conflict of interest noted during the conduct of this study. Confidentiality was maintained, and all participants verbally consented to participate in this research. This study is approved by the institutional Research Ethics Board.

\section{RESULT}

The survey response rate was $68 \%$, and all responses included in the analysis. Doctors who responded to the study were mostly between the age brackets of 36 to 45 years (47.1\%). $41.2 \%$ of them were Assistant Consultants. Most of the participants of the research were male accounting to $82.4 \%$ of the population. (Please see Table 1 for detail)

Table1. Demographic Data

\begin{tabular}{|l|l|l|}
\hline Variables & \multicolumn{1}{|c|}{ Category } & \multicolumn{1}{|c|}{$\mathbf{N}(\%)$} \\
\hline Age Group & $25-35$ Yrs. & $19(37.3 \%)$ \\
\cline { 2 - 3 } & $36-45$ Yrs & $24(47.1 \%)$ \\
\cline { 2 - 3 } & $46-55$ Yrs & $7(13.7 \%)$ \\
\cline { 2 - 3 } Specialty & 56 Yrs. & $1(2.0 \%)$ \\
\hline \multirow{5}{*}{ Gender } & Male & $42(82.4 \%)$ \\
\cline { 2 - 3 } & Female & $9(17.6 \%)$ \\
\cline { 2 - 3 } & Oncology & $16(31.4 \%)$ \\
\cline { 2 - 3 } & Hematology & $14(27.5 \%)$ \\
\cline { 2 - 3 } & Radiation & $5(9.8 \%)$ \\
\cline { 2 - 3 } & Oncology & \\
\cline { 2 - 3 } & Palliative Care & $10(19.6 \%)$ \\
\cline { 2 - 3 } & Pediatric & $6(11.8 \%)$ \\
& Hematology & \\
& Oncology & $7(13.7 \%)$ \\
\hline Job Title & Resident & $21(41.2 \%)$ \\
\cline { 2 - 3 } & Assistant & $1(2.0 \%)$ \\
\cline { 2 - 3 } & Fellow & $14(27.5 \%)$ \\
\cline { 2 - 3 } & Associate & \\
\cline { 2 - 3 } & Consultant & \\
\hline
\end{tabular}

The results indicated that issues about physicians' beliefs in resuscitation were varied. 
Physicians' View on Resuscitation Discussions among Patients and their Family in Comprehensive Cancer Center

The responding physicians divided in when discussions of resuscitation should occur, and more than half of them were depressed after such discussion. The majority of respondents (84\%) identified the discussion of resuscitation with patients and relatives as important. $57 \%$ $(n=29)$ stated that having both patients and relatives, together, is the preferred set-up when resuscitation is being discussed, and only $12 \%$ $(n=6)$ said that patients alone should be involved in this discussion.

The best time for resuscitation discussion is when the patient is conscious and oriented by $86 \%(n=43)$ of responders; $10.0 \%(n=5)$ stated this discussion should take place only when the patient is comatose, and requirement of resuscitative measures are imminent.

Almost all responders 98\% $(n=50)$ stated that resuscitation should be discouraged in uncontrolled or metastatic disease; one physician $(2.0 \%)$ reported that that aggressive resuscitation should be offered to all irrespective of stage or cause. Only 22 (43.1\%) were aware that there is a hospital protocol addressing the issue of resuscitation in terminal patients (Please see Table 2).

\section{DISCUSSION}

Discussing resuscitation with cancer patients and their relatives has remained a complex and challenging task. Identifying who among the patients need resuscitation is one of the most serious concerns in cancer care. From the data of this study, it has revealed that $5.9 \%$ respondents believe that patients with controlled primary or with acute and reversible causes must be the only ones to receive resuscitation.

Our study highlights that most physicians who responded believe that resuscitation should be discouraged in medically hopeless cases.

However, physician's knowledge about the information about any law for resuscitation and institutional policies remains very low, and this leads to a low level of legal awareness regarding resuscitation concerns which affect the oncology practitioners on a day to day basis.

Table2. Responses to Questions Addressing Issues Pertaining to Resuscitation in Terminal Cancer

Patients $\{$ Responses $($ Total $=51)\}$

\begin{tabular}{|c|c|c|}
\hline Variables & Category & $\mathbf{N}(\%)$ \\
\hline \multirow{3}{*}{$\begin{array}{l}\text { Do you feel } \\
\text { resuscitation } \\
\text { should be } \\
\text { discussed with } \\
\text { the patient or } \\
\text { relatives? }\end{array}$} & Yes & $43(84.3 \%)$ \\
\hline & No & $3(5.9 \%)$ \\
\hline & Not Sure & $5(9.8 \%)$ \\
\hline \multirow{3}{*}{$\begin{array}{l}\text { If Yes, should } \\
\text { it be } \\
\text { discussed? }\end{array}$} & with patient & $6(11.8 \%)$ \\
\hline & with relatives & $16(31.4 \%)$ \\
\hline & $\begin{array}{l}\text { with both patient } \\
\text { and relatives }\end{array}$ & $29(56.9 \%)$ \\
\hline \multirow{3}{*}{$\begin{array}{l}\text { If Yes, When } \\
\text { is the time to } \\
\text { discuss the } \\
\text { about the } \\
\text { resuscitation? }\end{array}$} & $\begin{array}{l}\text { When the patient } \\
\text { is still conscious, } \\
\text { oriented }\end{array}$ & $43(86.0 \%)$ \\
\hline & $\begin{array}{l}\text { When the patient } \\
\text { is comatose and } \\
\text { requirement for } \\
\text { resuscitation is } \\
\text { imminent }\end{array}$ & $5(10.0 \%)$ \\
\hline & $\begin{array}{l}\text { Along with the } \\
\text { resuscitation } \\
\text { effort }\end{array}$ & $2(4.0 \%)$ \\
\hline \multirow{3}{*}{\begin{tabular}{|l|} 
In which \\
situation \\
aggressive \\
resuscitation \\
in cancer \\
patients is not \\
warranted?
\end{tabular}} & \begin{tabular}{ll}
\multicolumn{2}{l}{ Controlled disease } \\
or $\quad$ reversible \\
causes
\end{tabular} & $3(5.9 \%)$ \\
\hline & $\begin{array}{l}\text { Uncontrolled or } \\
\text { metastatic disease }\end{array}$ & $47(92.2 \%)$ \\
\hline & $\begin{array}{l}\text { Will attempt } \\
\text { resuscitation in all } \\
\text { cancer patients } \\
\text { regardless of } \\
\text { stage/cause will } \\
\text { attempt } \\
\text { resuscitation }\end{array}$ & $1(2.0 \%)$ \\
\hline \multirow{3}{*}{$\begin{array}{l}\text { Is there any } \\
\text { law regarding } \\
\text { resuscitation } \\
\text { in cancer } \\
\text { patients? } \\
\end{array}$} & Yes & $22(43.1 \%)$ \\
\hline & No & $6(11.8 \%)$ \\
\hline & Not Sure & $23(45.1 \%)$ \\
\hline
\end{tabular}

As mentioned from previous research, attitudes and behaviors of professional caregivers may vary widely, as they can be affected by several factors, such as conscience, religion, culture, personal experience, and education [4, 14, 15]. While this may be true, physicians must extend their efforts in providing disclosure about patient's resuscitation status in a matter that 
Physicians' View on Resuscitation Discussions among Patients and their Family in Comprehensive Cancer Center

suffices their needs about the explanation of its information.

It is important for physicians to inform patient and their families about desired outcomes of $\mathrm{CPR}$ and its potential risk factors. Also, if patient condition indicates, and if the need arises the option for DNR should be discussed with them. It is vital that families should actively participate in the decision-making. There are ethical and moral concerns that need consideration with resuscitation and the DNR concept.

The study shows that the majority wish to have DNR discussion should be made early led by the consultants attending to the patients. We believe that inappropriate resuscitations add stress to the 'code team' consisting of junior doctors who would call if the patient arrests. We also hope to implement simulation training for DNR discussion to provide physicians some practice and increase their comfort levels.

\section{CONCLUSION}

Resuscitation remains a challenging area with disagreements on patient's involvement and final decision making. As observed in the result of this study, physicians need to be more informed of hospital laws regarding resuscitation in cancer patients. In this way, they will be more guided by the regulations provided for them. Additional training may be probably included so as to provide a supplemental strategy on how physicians may have the knowledge and at the same time, for them to adhere to the disclosure of resuscitation for patients.

\section{REFERENCES}

[1] Fallahi, M., Banaderakhshan, H., Abdi, A., Borhani, F., Kaviannezhad, R., \& Karimpour, H. A. (2016). The Iranian physicians attitude toward the do not resuscitate order. Journal of Multidisciplinary Healthcare, 9, 279.

[2] Morgan, R., \& Westmoreland, C. (2002). Survey of junior hospital doctors' attitudes to cardiopulmonary resuscitation. Postgraduate medical journal, 78(921), 413-415.

[3] Fontana D, Abouserie R. Stress levels, gender and personality, factors in teachers. Br J Educ Psychol 1993;63:261-70.

[4] Nikolaou, N. I., Patialiakas, A., Kokkinos, P., Christou, A. H., Chasikides, C., Tasouli, A., ... \& Papafanis, T. (2014). Attitudes of Healthcare Professionals Involved in Cardiology Practice Towards Key Points of Contemporary Guidelines on Resuscitation. Hellenic J Cardiol, 55, 378-385.

[5] Baird, G., Sammy, I., Nunes, P., \& Paul, J. (2013). Attitudes and practices regarding resuscitation in emergency departments in Trinidad and Tobago. Emergency Medicine Journal, emermed-2012.

[6] Wenger, N. S., Phillips, R. S., Teno, J. M., Oye, R. K., Dawson, N. V., Liu, H., ... \& Lynn, J. (2000). Physician understanding of patient resuscitation preferences: insights and clinical implications. Journal of the American Geriatrics Society, 48(S1), S44-S51.

[7] Mian, P., Warchal, S., Whitney, S., Fitzmaurice, J., \& Tancredi, D. (2007). Impact of a multifaceted intervention on nurses' and physicians' attitudes and behaviors toward family presence during resuscitation. Critical Care Nurse, 27(1), 52-61.

[8] Holland, J. C., Holland, J. C., Geary, N., Marchini, A., \& Tross, S. (1987). Psychosocial Issues: an international survey of physician attitudes and practice in regard to revealing the diagnosis of cancer. Cancer investigation, 5(2), 151-154.

[9] Hilden, H. M., Louhiala, P., \& Palo, J. (2004). End of life decisions: attitudes of Finnish physicians. Journal of medical ethics, 30(4), 362-365.

[10] Miller, D. L., Jahnigen, D. W., Gorbien, M. J., \& Simbartl, L. (1992). Cardiopulmonary Resuscitation: How Useful?: Attitudes and Knowledge of an Elderly Population. Archives of Internal Medicine, 152(3), 578-582.

[11] Blackhall, L. J., Frank, G., Murphy, S. T., Michel, V., Palmer, J. M., \& Azen, S. P. (1999). Ethnicity and attitudes towards life sustaining technology. Social science \& medicine, 48(12), 1779-1789.

[12] Kelly, W. F., Eliasson, A. H., Stocker, D. J., \& Hnatiuk, W. (2002). Do specialists differ on donot-resuscitate decisions? CHEST Journal, 121(3), 957-963.

[13] Bruera, E., Neumann, C. M., Mazzocato, C., Stiefel, F., \& Sala, R. (2000). Attitudes and beliefs of palliative care physicians regarding communication with terminally ill cancer patients. Palliative medicine, 14(4), 287-298. 
Physicians' View on Resuscitation Discussions among Patients and their Family in Comprehensive Cancer Center

[14] Bowman, S. M., Major, D. A., \& Green, W. P. (1984). Cardiopulmonary resuscitation (CPR): patient factors and decision making. Archives of internal medicine, 144(11), 2229-2232.
[15] Varon, J., Fromm, R. E., Sternbach, G. L., \& Combs, A. H. (1993). Discrepancy in resuscitation beliefs among physicians at various levels of training. The American journal of emergency medicine, 11(3), 290-292.

Citation: Hamdan A, AlShammary S, Tamani J, Sashidharan S, Ratnapalan S, AlHarbi M. Physicians'View on Resuscitation Discussions among Patients and their Family in Comprehensive Cancer Center. International Journal of Research Studies in Medical and Health Sciences. 2017;2(5):11-16.

Copyright: (C) 2017 Hamdan A, et al. This is an open-access article distributed under the terms of the Creative Commons Attribution License, which permits unrestricted use, distribution, and reproduction in any medium, provided the original author and source are credited. 